Pacific

Journal of

Mathematics

ASYMPTOTIC BEHAVIOR OF POSITIVE SOLUTIONS OF SOME ELLIPTIC PROBLEMS

E.N. DANCER, Yihong Du, AND Li Ma 


\title{
ASYMPTOTIC BEHAVIOR OF POSITIVE SOLUTIONS OF SOME ELLIPTIC PROBLEMS
}

\author{
E.N. Dancer, Yihong Du, And Li Ma
}

\begin{abstract}
In this paper, we discuss the asymptotic behavior of the positive solutions of the problem $-\Delta u=a u-b u^{p},\left.u\right|_{\partial \Omega}=0$ as $p \rightarrow 1+0$ and as $p \rightarrow \infty$. We show that, for each case, the behavior is determined by a limiting problem. Moreover, the limiting problem is of free boundary nature when $p \rightarrow \infty$.
\end{abstract}

\section{Introduction and main results.}

In this paper, we study the asymptotic behavior of positive solutions of the problem

$$
-\Delta u=a u-b(x) u^{p}, x \in \Omega ; u=0, x \in \partial \Omega,
$$

for $p$ near 1 and near $\infty$, respectively. Here $\Omega$ is a bounded smooth domain in $R^{N}(N \geq 1)$ and $b(x)$ is a nonnegative function in $C(\bar{\Omega}), a$ and $p$ are constants but the exponent $p$ is always greater than 1 .

Problem (1.1) arises from mathematical biology and Riemannian geometry, and has attracted considerable interests; see, for example, $[\mathbf{A T}],[\mathbf{A M}]$, $[\mathbf{D}],[\mathbf{d P}],[\mathbf{D H}],[\mathbf{F K L M}],[\mathbf{K W}],[\mathbf{M}]$ and $[\mathbf{O}]$. The dependence of the positive solutions of (1.1) on the parameter $a$ is well understood but little is known about the dependence on $p$.

When $b(x)$ is strictly positive on $\Omega,(1.1)$ is the steady-state logistic equation and it is well-known that for fixed $p>1$ it has no positive solution if $a \leq \lambda_{1}^{\Omega}$ and there is a unique positive solution $u=u_{a}$ when $a>\lambda_{1}^{\Omega}$, where $\lambda_{1}^{\Omega}$ denotes the first eigenvalue of the problem

$$
-\Delta u=\lambda u,\left.u\right|_{\partial \Omega}=0 .
$$

Moreover, $a \rightarrow u_{a}$ is continuous and strictly increasing as a function from $\left(\lambda_{1}^{\Omega}, \infty\right)$ to $C(\bar{\Omega})$ (with the natural order), and

$$
\lim _{a \rightarrow \lambda_{1}^{\Omega}+0} u_{a}(x)=0 \text { uniformly in } \bar{\Omega} \text {; }
$$

$\lim _{a \rightarrow \infty} u_{a}(x)=\infty$ uniformly on any compact subset of $\Omega$.

When $b^{-1}(0):=\{x \in \Omega: b(x)=0\}$ is a proper subset of $\Omega$, the behavior of (1.1) is more complicated. Assume for simplicity that $b^{-1}(0)=\bar{\Omega}_{0} \subset \subset \Omega$, 
where $\Omega_{0}$ is open, connected and with smooth boundary. Then it is wellknown that (1.1) has no positive solution unless $a \in\left(\lambda_{1}^{\Omega}, \lambda_{1}^{\Omega_{0}}\right)$, in which case there is a unique positive solution $u_{a}$ which varies continuously with $a$ and is strictly increasing in $a$. Moreover, $u_{a} \rightarrow 0$ uniformly on $\bar{\Omega}$ as $a \rightarrow \lambda_{1}^{\Omega}+0$, but as $a \rightarrow \lambda_{1}^{\Omega_{0}}, u_{a}(x) \rightarrow \infty$ uniformly on $\bar{\Omega}_{0}$ and $u_{a} \rightarrow U$ uniformly on any compact subset of $\bar{\Omega} \backslash \bar{\Omega}_{0}$, where $U$ is the unique minimal positive solution of the boundary blow-up problem

$$
-\Delta u=a u-b(x) u^{p}, x \in \Omega \backslash \bar{\Omega}_{0} ;\left.u\right|_{\partial \Omega}=0,\left.u\right|_{\partial \Omega_{0}}=\infty .
$$

We refer to $[\mathbf{D H}]$ and the references therein for more details.

To understand the effect of the exponent $p$ on the unique positive solution of (1.1), we fix $a$ and consider the extreme cases, that is when $p \rightarrow 1+0$ and when $p \rightarrow \infty$. In each case, we obtain a limiting problem which determines the asymptotical behavior of (1.1).

To describe our results, we need to recall several simple properties of the first eigenvalue of the Laplacian operator. Let $\phi \in L^{\infty}(\Omega)$ and denote by $\lambda_{1}^{\Omega}(\phi)$ the first eigenvalue of the problem

$$
-\Delta u+\phi u=\lambda u,\left.u\right|_{\partial \Omega}=0 .
$$

Clearly, $\lambda_{1}^{\Omega}(0)=\lambda_{1}^{\Omega}$. It is well-known that $\lambda_{1}^{\Omega}\left(\phi_{n}\right) \rightarrow \lambda_{1}^{\Omega}(\phi)$ whenever $\phi_{n} \rightarrow \phi$ in $L^{\infty}(\Omega)$, and when $\phi \leq \psi$ but $\phi \not \equiv \psi$ in $\Omega$, then $\lambda_{1}^{\Omega}(\phi)<\lambda_{1}^{\Omega}(\psi)$. It follows easily that, when $b(x) \geq \delta>0$ on $\Omega$, then $\lambda(\alpha):=\lambda_{1}^{\Omega}(\alpha b)$ is a strictly increasing function with $\lambda(0)=\lambda_{1}^{\Omega}$ and $\lambda(\alpha) \rightarrow \infty$ as $\alpha \rightarrow \infty$. Therefore, for any given $a>\lambda_{1}^{\Omega}$, there is a unique $\alpha>0$ such that

$$
a=\lambda_{1}^{\Omega}(\alpha b) \text {. }
$$

We denote by $U_{\alpha}$ the corresponding positive normalized eigenfunction:

$$
-\Delta U_{\alpha}+\alpha b U_{\alpha}=a U_{\alpha}, U_{\alpha}>0,\left.U_{\alpha}\right|_{\partial \Omega}=0,\left\|U_{\alpha}\right\|_{\infty}=1 .
$$

Here and in what follows, we use the notation $\|\cdot\|_{\infty}=\|\cdot\|_{L^{\infty}(\Omega)}$.

When $b^{-1}(0)=\bar{\Omega}_{0}$ is not empty, we assume as before that $\Omega_{0} \subset \subset \Omega$ is open, connected and with smooth boundary. Then $\lambda(\alpha)=\lambda_{1}^{\Omega}(\alpha b)$ is still strictly increasing with $\lambda(0)=\lambda_{1}^{\Omega}$, but (see $[\mathbf{D}]$ and $[\mathbf{F K L M}]$ )

$$
\lim _{\alpha \rightarrow \infty} \lambda(\alpha)=\lambda_{1}^{\Omega_{0}}
$$

Thus for any given $a \in\left(\lambda_{1}^{\Omega}, \lambda_{1}^{\Omega_{0}}\right)$, there is a unique $\alpha>0$ satisfying (1.2) which determines a unique $U_{\alpha}$ through (1.3).

We are now ready to state our main results.

Theorem 1.1. Suppose that $b(x)>0$ on $\bar{\Omega}$ and $a>\lambda_{1}^{\Omega}$. Let $u_{p}$ denote the unique positive solution of (1.1). Then the following results hold: 
(i) When $a<\lambda_{1}^{\Omega}(b)$, we have $u_{p} \rightarrow 0$ uniformly on $\bar{\Omega}$ as $p \rightarrow 1+0$. Moreover, as $p \rightarrow 1+0$,

$$
(p-1) \ln \left\|u_{p}\right\|_{\infty} \rightarrow \ln \alpha, u_{p} /\left\|u_{p}\right\|_{\infty} \rightarrow U_{\alpha} \text { in } C^{1}(\bar{\Omega}),
$$

where $\alpha$ and $U_{\alpha}$ are determined by (1.2) and (1.3), respectively.

(ii) When $a>\lambda_{1}^{\Omega}(b)$, we have $u_{p} \rightarrow \infty$ uniformly on any compact subset of $\Omega$ as $p \rightarrow 1+0$. Moreover, (1.4) holds.

(iii) When $a=\lambda_{1}(b, \Omega)$, we have $u_{p} \rightarrow c U_{1}$ in $C^{1}(\bar{\Omega})$ as $p \rightarrow 1+0$, where $U_{1}$ is given by (1.3) with $\alpha=1$ and

$$
c=\exp \left(\int_{\Omega} b U_{1}^{2} \ln U_{1} d x / \int_{\Omega} b U_{1}^{2} d x\right) .
$$

To understand the case that $p \rightarrow \infty$, we need the following free boundary problem:

$$
-\Delta w=a \chi_{\{w<1\}} w, w>0,\left.w\right|_{\partial \Omega}=0,\|w\|_{\infty}=1,
$$

which also arises as a limiting problem for the degenerate predator-prey model (see [DD2]). The following result has been proved in [DD2]:

Proposition 1.2. For any $a \geq \lambda_{1}^{\Omega}$, (1.5) has a unique weak solution, and when $a<\lambda_{1}^{\Omega}$, (1.5) has no solution.

With the help of Proposition 1.2, we will prove the following:

Theorem 1.3. Suppose that $b(x)>0$ on $\bar{\Omega}$ and $a>\lambda_{1}^{\Omega}$. Let $u_{p}$ denote the unique positive solution of (1.1). Then $u_{p} \rightarrow v$ in $C^{1}(\bar{\Omega})$ as $p \rightarrow \infty$, where $v$ is the unique positive weak solution of (1.5).

When $\bar{\Omega}_{0}:=b^{-1}(0)$ is a nontrivial subset of $\Omega$, it turns out that the techniques in proving Theorems 1.1 and 1.3 are not enough. One new ingredient for dealing with this case is the following result obtained in [DD1, Lemma $2.2]$ :

Lemma 1.4. Suppose that $\left\{u_{n}\right\} \subset C^{1}(\bar{\Omega})$ satisfies (in the weak sense) for some positive constant $\lambda$,

$$
-\Delta u_{n} \leq \lambda u_{n}, u_{n} \geq 0 \text { in } \Omega ;\left.u_{n}\right|_{\partial \Omega}=0,\left\|u_{n}\right\|_{\infty}=1 .
$$

Then it has a subsequence converging weakly in $H_{0}^{1}(\Omega)$ and strongly in $L^{q}(\Omega)$ for any $q \geq 1$, to some $u$ with $\|u\|_{\infty}=1$.

Theorem 1.5. Suppose that $\bar{\Omega}_{0}=b^{-1}(0)$ has nonempty interior which is connected with smooth boundary and $\Omega_{0} \subset \subset \Omega$. Let $a \in\left(\lambda_{1}^{\Omega}, \lambda_{1}^{\Omega_{0}}\right)$ and denote by $u_{p}$ the unique positive solution of (1.1). Then the conclusions (i)-(iii) in Theorem 1.1 hold.

As Theorem 1.5 concludes that when $b^{-1}(0) \neq \varnothing$ and $p \rightarrow 1$, the behavior of $u_{p}$ is the same as when $b^{-1}(0)=\varnothing$, it is tempting to think that this is also the case when $p \rightarrow \infty$. It turns out, however, this is not true. 
Theorem 1.6. Suppose that $\bar{\Omega}_{0}=b^{-1}(0)$ has nonempty interior which is connected with smooth boundary and $\Omega_{0} \subset \subset \Omega$. Let $a \in\left(\lambda_{1}^{\Omega}, \lambda_{1}^{\Omega_{0}}\right)$ and denote by $u_{p}$ the unique positive solution of (1.1). Suppose $p_{n} \rightarrow \infty$ and denote $u_{n}=u_{p_{n}}$. Then, subject to a subsequence, $u_{n} \rightarrow u$ in $L^{q}(\Omega)$ for all $q \geq 1$, where $u \in K$ is a nontrivial nonnegative solution of the following variational inequality:

$$
\begin{gathered}
\int_{\Omega} \nabla u \cdot \nabla(v-u) d x-\int_{\Omega} a u(v-u) d x \geq 0, \forall v \in K, \\
K:=\left\{w \in H_{0}^{1}(\Omega): w \leq 1 \text { a.e. in } \Omega \backslash \Omega_{0}\right\} .
\end{gathered}
$$

In a forthcoming paper ([DD3]), we will show that (1.6) has a unique positive solution for $a \in\left(\lambda_{1}^{\Omega}, \lambda_{1}^{\Omega_{0}}\right)$, and hence $u_{p} \rightarrow u$ as $p \rightarrow \infty$ in $L^{q}(\Omega)$. Let us note that (1.6) is different from (1.5). In fact, it has been shown in [DD2] that (1.5) is equivalent to the variational inequality

$$
\begin{gathered}
\int_{\Omega} \nabla u \cdot \nabla(v-u) d x-\int_{\Omega} a u(v-u) d x \geq 0, \forall v \in K_{0}, \\
K_{0}:=\left\{w \in H_{0}^{1}(\Omega): w \leq 1 \text { a.e. in } \Omega\right\} .
\end{gathered}
$$

Moreover, it is possible to show that for any given compact subset $D$ of $\Omega$, there exists a large $a_{D}$ such that the unique solution of (1.5) satisfies $w=1$ on $D$ when $a>a_{D}$. (More precise results are discussed briefly in [DD2].) It is easily seen that for such $a$, and for those $\Omega_{0} \subset D$ satisfying $\lambda_{1}^{\Omega_{0}}>a$, if we let $u=w$ on $\bar{\Omega} \backslash \Omega_{0}$; and on $\Omega_{0}$, let $u$ equal the unique solution to $-\Delta u=a u,\left.u\right|_{\partial \Omega_{0}}=1$, then $u$ solves (1.6).

Remark 1.7. From our proofs, it is easy to see that our assumptions on the smoothness of $\partial \Omega_{0}$ can be considerably weakened. For example, all our main results hold if $\Omega_{0}$ only has Lipschitz boundary.

Remark 1.8. If $b^{-1}(0)$ consists of a single point in $\Omega$, then Theorems 1.5 and 1.6 reduce to Theorems 1.1 and 1.3 , respectively. This follows easily by checking the proofs. We intend to further consider the case that $b^{-1}(0)$ has measure zero in [DD3].

The rest of the paper consists of the proofs of our results given above. Theorem 1.1 is proved in Section 2; Theorems 1.3 and 1.5 are proved in Sections 3 and 4, respectively; Section 5 gives the proof of Theorem 1.6. The main techniques involved are various elliptic estimates and comparison principles. Several results and techniques from [DD2] will be used, including fine properties of functions in Sobolev spaces and the use of variational inequalities. 


\section{Proof of Theorem 1.1.}

Set $M_{p}=\left\|u_{p}\right\|_{\infty}=\max _{\bar{\Omega}} u_{p}$. Then it is clear that the maximum is achieved in the interior of the domain $\Omega$, say at $x_{p} \in \Omega$. Using the equation for $u_{p}$ at the maximum point $x=x_{p}$ we have

$$
a M_{p}-b\left(x_{p}\right) M_{p}^{p} \geq 0 .
$$

Hence,

$$
M_{p}^{p-1} \leq a / \min _{\bar{\Omega}} b .
$$

To understand the asymptotic behaviour of $u_{p}$ as $p \rightarrow 1+0$, we choose an arbitrary sequence $p_{n} \rightarrow 1+0$ and use the notation

$$
u_{n}=u_{p_{n}}, M_{n}=M_{p_{n}}, \alpha_{n}=M_{p_{n}}^{p_{n}-1}, w_{n}=u_{n} / M_{n}
$$

Clearly $w_{n}$ satisfies

$$
-\Delta w_{n}=a w_{n}-\alpha_{n} b w_{n}^{p_{n}},\left.w_{n}\right|_{\partial \Omega}=0 .
$$

From (2.1) one sees that the right-hand side of (2.3) has a bound in $L^{\infty}(\Omega)$ which is independent of $n$. Thus, by standard elliptic estimates, $\left\{w_{n}\right\}$ is bounded in $W^{2, q}(\Omega)$ for any $q>1$. By the Sobolev imbedding theorem, this implies that this sequence is compact in $C^{1}(\bar{\Omega})$. Therefore, subject to a subsequence, $w_{n} \rightarrow w$ in $C^{1}(\bar{\Omega})$. We may also assume that $\alpha_{n} \rightarrow \alpha$. Then from (2.3) we obtain, in the weak sense,

$$
-\Delta w=(a-\alpha b) w,\left.w\right|_{\partial \Omega}=0 .
$$

As $w$ is nonnegative with $\|w\|_{\infty}=1$, we necessarily have $a=\lambda_{1}^{\Omega}(\alpha b)$ and hence $\alpha$ is uniquely determined by (1.2) and $w=U_{\alpha}$ given by (1.3). This implies that $\alpha_{n} \rightarrow \alpha$ and $w_{n} \rightarrow U_{\alpha}$ hold for the entire original sequences. Therefore, we have proved that $M_{p}^{p-1} \rightarrow \alpha$ and $u_{p} / M_{p} \rightarrow U_{\alpha}$ in $C^{1}(\bar{\Omega})$ as $p \rightarrow 1+0$. This shows the validity of (1.4).

When $a<\lambda_{1}^{\Omega}(b)$, we must have $\alpha \in(0,1)$ and it follows from

$$
\lim _{p \rightarrow 1+0}(p-1) \ln M_{p}=\ln \alpha
$$

that $M_{p} \rightarrow 0$ as $p \rightarrow 1+0$. This proves Part (i) of Theorem 1.1.

When $a>\lambda_{1}^{\Omega}(b)$, we must have $\alpha>1$ and it follows from (2.4) that $M_{p} \rightarrow \infty$ as $p \rightarrow 1+0$. To prove Part (ii) of Theorem 1.1, it remains to show that as $p \rightarrow 1+0, u_{p}(x) \rightarrow \infty$ uniformly on any compact subset of $\Omega$. To this end, for any given large number $\beta$, we define $V=\beta U_{\alpha}$ and obtain

$$
\Delta V+a V-b V^{p}=b\left(\alpha V-V^{p}\right) .
$$

For those $x$ where $V(x) \leq 1, \alpha V-V^{p} \geq(\alpha-1) V \geq 0$; on the set $\{x \in \Omega$ : $V(x) \geq 1$, since $V^{p} \rightarrow V$ uniformly as $p \rightarrow 1$, and since $\alpha V-V \geq \alpha-1>0$, we can find $\epsilon=\epsilon(\beta)>0$ small enough such that $\alpha V-V^{p}>0$ for all $p \in(1,1+\epsilon)$. Thus, for $p \in(1,1+\epsilon), V$ is a lower solution to (1.1). As 
any large positive constant is an upper solution of (1.1), its unique positive solution $u_{p}$ must satisfy $u_{p} \geq V=\beta U_{\alpha}$. This implies that as $p \rightarrow 1+0$, $u_{p} \rightarrow \infty$ uniformly on any compact subset of $\Omega$ and Part (ii) of Theorem 1.1 is proved.

We consider now the case that $a=\lambda_{1}^{\Omega}(b)$. We have $\alpha=1$ and hence cannot draw a conclusion for $\lim _{p \rightarrow 1+0} M_{p}$ from (2.4). Denote $w_{p}=u_{p} / M_{p}$. We have

$$
-\Delta w_{p}=a w_{p}-b M_{p}^{p-1} w_{p}^{p},\left.w_{p}\right|_{\partial \Omega}=0 .
$$

Multiply this equation by $U_{1}$, which is given by (1.3) with $\alpha=1$, and integrate by parts. It results

$$
\int_{\Omega}(a-b) U_{1} w_{p} d x=\int_{\Omega}\left(a w_{p}-b M_{p}^{p-1} w_{p}^{p}\right) U_{1} d x .
$$

Hence

$$
\int_{\Omega} b\left(w_{p}-M_{p}^{p-1} w_{p}^{p}\right) U_{1} d x=0
$$

and

$$
\int_{\Omega} \frac{M_{p}^{p-1}-1}{p-1} b w_{p}^{p} U_{1} d x=\int_{\Omega} \frac{1-w_{p}^{p-1}}{p-1} b w_{p} U_{1} d x .
$$

Since $w_{p} \rightarrow U_{1}$ as $p \rightarrow 1+0$ in $C^{1}(\bar{\Omega})$, and by the Hopf boundary lemma, $\partial U_{1} / \partial \nu<0$ on $\partial \Omega$, we obtain $w_{p} / U_{1} \rightarrow 1$ uniformly on $\bar{\Omega}$. It follows that

$$
\left\|\ln w_{p}-\ln U_{1}\right\|_{L^{\infty}(\Omega)}=o(1)
$$

as $p \rightarrow 1+0$. Therefore,

$$
\frac{1-w_{p}^{p-1}}{p-1} w_{p}=\frac{1-e^{(p-1)\left(\ln U_{1}+o(1)\right)}}{p-1} w_{p} \rightarrow U_{1} \ln U_{1}
$$

uniformly on $\bar{\Omega}$ as $p \rightarrow 1+0$. From this, we see immediately that the right-hand side of $(2.5)$ converges to

$$
\int_{\Omega} b U_{1}^{2} \ln U_{1} d x
$$

Thus,

$$
\lim _{p \rightarrow 1+0} \int_{\Omega} \frac{M_{p}^{p-1}-1}{p-1} b w_{p}^{p} U_{1} d x=\int_{\Omega} b U_{1}^{2} \ln U_{1} d x
$$

and

$$
\lim _{p \rightarrow 1+0} \frac{M_{p}^{p-1}-1}{p-1}=\int_{\Omega} b U_{1}^{2} \ln U_{1} d x / \int_{\Omega} b U_{1}^{2} d x .
$$

We show next that

$$
c:=\lim _{p \rightarrow 1+0} M_{p}
$$


exists and is uniquely determined by

$$
\ln c=\int_{\Omega} b U_{1}^{2} \ln U_{1} d x / \int_{\Omega} b U_{1}^{2} d x .
$$

We first claim that

$$
M_{*}:=\varliminf_{p \rightarrow 1+0} M_{p}>0, M^{*}:=\varlimsup_{p \rightarrow 1+0} M_{p}<\infty .
$$

Otherwise, we can find a sequence $p_{n} \rightarrow 1+0$ such that $M_{n}:=M_{p_{n}} \rightarrow 0$ or $M_{n} \rightarrow \infty$. In the former case, we deduce, for all large $n$,

$$
\frac{M_{n}^{p_{n}-1}-1}{p_{n}-1} \leq \frac{\epsilon^{p_{n}-1}-1}{p_{n}-1} \rightarrow \ln \epsilon
$$

as $n \rightarrow \infty$, for any given $\epsilon>0$. This leads to a contradiction to (2.6). In the latter case, we obtain, for all large $n$,

$$
\frac{M_{n}^{p_{n}-1}-1}{p_{n}-1} \geq \frac{M^{p_{n}-1}-1}{p_{n}-1} \rightarrow \ln M
$$

as $n \rightarrow \infty$, for any given $M>0$. This also leads to a contradiction to (2.6). Thus, $0<M_{*} \leq M^{*}<\infty$. For any given small $\epsilon>0$, a similar argument to the above leads to

$$
\begin{aligned}
& \ln \left(M_{*}+\epsilon\right) \geq \int_{\Omega} b U_{1}^{2} \ln U_{1} d x / \int_{\Omega} b U_{1}^{2} d x \\
& \ln \left(M^{*}-\epsilon\right) \leq \int_{\Omega} b U_{1}^{2} \ln U_{1} d x / \int_{\Omega} b U_{1}^{2} d x .
\end{aligned}
$$

Thus we necessarily have

$$
M_{*}=M^{*}=c=\exp \left(\int_{\Omega} b U_{1}^{2} \ln U_{1} d x / \int_{\Omega} b U_{1}^{2} d x\right),
$$

and $u_{p} \rightarrow c U_{1}$ as $p \rightarrow 1+0$ in $C^{1}(\bar{\Omega})$. This finishes the proof of Theorem 1.1.

\section{Proof of Theorem 1.3.}

We clearly still have (2.1). Let $p_{n}$ be a sequence converging to $\infty$ and use the notations in (2.2). We find that $w_{n}$ satisfies (2.3) whose right-hand side has a bound in $L^{\infty}(\Omega)$ which is independent of $n$. Thus, as in Section 2, subject to a subsequence, $w_{n} \rightarrow w$ in $C^{1}(\bar{\Omega})$.

The equation satisfied by $w_{n}$ can also be written as

$$
-\Delta w_{n}=a w_{n}-b u_{n}^{p_{n}-1} w_{n},\left.w_{n}\right|_{\partial \Omega}=0 .
$$

From (2.1) we deduce

$$
0 \leq u_{n}^{p_{n}-1} \leq a / \min _{\bar{\Omega}} b
$$


Hence, by passing to a subsequence, we may assume that $b u_{n}^{p_{n}-1} \rightarrow \psi$ weakly in $L^{2}(\Omega)$. Clearly we must have $0 \leq \psi \leq\|b\|_{\infty} a / \min _{\bar{\Omega}} b$. Passing to the weak limit in (3.1) we find that $w$ is a nontrivial weak solution to the problem

$$
-\Delta w=(a-\psi) w,\left.w\right|_{\partial \Omega}=0 .
$$

As $a-\psi \in L^{\infty}(\Omega)$, it follows from the Harnack inequality that $w(x)>0$ in $\Omega$.

From (2.1) we obtain

$$
M_{n} \leq\left(a / \min _{\bar{\Omega}} b\right)^{1 /\left(p_{n}-1\right)} \rightarrow 1 \text { as } n \rightarrow \infty .
$$

It follows that $\varlimsup_{n \rightarrow \infty} M_{n} \leq 1$. If $\underline{\lim }_{n \rightarrow \infty} M_{n}<1$, then by passing to a subsequence, we may assume that $M_{n} \leq 1-\epsilon$ for all $n$ and some $\epsilon>0$. It follows then $u_{n}^{p_{n}-1} \leq(1-\epsilon)^{p_{n}-1} \rightarrow 0$ as $n \rightarrow \infty$. Hence $\psi=0$ and $w$ is a positive solution to $-\Delta w=a w,\left.w\right|_{\partial \Omega}=0$. This implies that $a=\lambda_{1}^{\Omega}$, contradicting our assumption that $a>\lambda_{1}^{\Omega}$. Thus we have proved that $M_{n} \rightarrow 1$ as $n \rightarrow \infty$. It follows that $u_{n} \rightarrow w$ in $C^{1}(\bar{\Omega})$.

Let $\Omega_{1}:=\{x \in \Omega: w(x)<1\}$. Then for any $x \in \Omega_{1}$, we can find $\delta>0$ such that $u_{n}(x)<1-\delta$ for all large $n$. It follows that $0 \leq u_{n}(x)^{p_{n}-1} \leq$ $(1-\delta)^{p_{n}-1} \rightarrow 0$ as $n \rightarrow \infty$. Thus we must have $\psi=0$ a.e. on $\Omega_{1}$. On the rest of $\Omega, w=1$ and we necessarily have $\Delta w=0$. (Here we regard $w$ as a member of $W^{2, q}(\Omega), q>1$.) Thus from (3.2), we deduce $\psi=a$ a.e. on $\Omega \backslash \Omega_{1}$. Therefore, $w$ satisfies

$$
-\Delta w=a \chi_{\{w<1\}} w, w>0,\left.w\right|_{\partial \Omega}=0,\|w\|_{\infty}=1 .
$$

By Proposition 1.2, problem (3.3) has a unique solution $v$. Hence $u_{n} \rightarrow v$ in $C^{1}(\bar{\Omega})$ for the entire original sequence. This implies that $u_{p} \rightarrow v$ in $C^{1}(\bar{\Omega})$ as $p \rightarrow \infty$. The proof of Theorem 1.3 is complete.

\section{Proof of Theorem 1.5.}

We will mainly follow the lines of the proof of Theorem 1.1. The main difficulty is that the estimate (2.1) is of no use anymore and therefore it is unclear whether $\left\{\alpha_{n}\right\}$ is still bounded. We will use Lemma 1.4 to overcome this difficulty.

Let $p_{n}$ be an arbitrary sequence of numbers converging to $1+0$. We employ the notations in (2.2) and find that $w_{n}$ meets the conditions in Lemma 1.4. Hence, by passing to a subsequence, we may assume that $w_{n} \rightarrow w$ weakly in $H_{0}^{1}(\Omega)$, strongly in $L^{q}(\Omega)$ for any $q \geq 1$, and $\|w\|_{\infty}=1$.

We claim that $\left\{\alpha_{n}\right\}$ is bounded. Otherwise, by passing to a subsequence, we may assume that $\alpha_{n} \rightarrow \infty$. Now we multiply (2.3), the equation satisfied by $w_{n}$, by $\phi / \alpha_{n}$ with $\phi \in C_{0}^{\infty}(\Omega)$ and integrate by parts. We obtain

$$
\left(\alpha_{n}\right)^{-1} \int_{\Omega} w_{n}(-\Delta \phi) d x=\left(\alpha_{n}\right)^{-1} \int_{\Omega} a w_{n} \phi d x-\int_{\Omega} b w_{n}^{p_{n}} \phi d x .
$$


Letting $n \rightarrow \infty$, we deduce

$$
\int_{\Omega} b w \phi d x=0
$$

As $\phi$ is arbitrary, this implies that $b w=0$ in $\Omega$. Hence, $w=0$ on $\Omega \backslash \Omega_{0}$. Since $w \in H_{0}^{1}(\Omega)$ and $\partial \Omega_{0}$ is smooth, this implies that $\left.w\right|_{\Omega_{0}} \in H_{0}^{1}\left(\Omega_{0}\right)$. Multiplying the equation for $w_{n}$ by an arbitrary $\phi \in C_{0}^{\infty}\left(\Omega_{0}\right)$ and integrating by parts, we obtain

$$
\int_{\Omega_{0}} \nabla w_{n} \cdot \nabla \phi d x=\int_{\Omega_{0}} a w_{n} \phi d x
$$

Passing to $n \rightarrow \infty$ we obtain

$$
\int_{\Omega_{0}} \nabla w \cdot \nabla \phi d x=\int_{\Omega_{0}} a w \phi d x .
$$

Thus $\left.w\right|_{\Omega_{0}}$ is a weak solution of the problem

$$
-\Delta u=a u,\left.u\right|_{\partial \Omega_{0}}=0 .
$$

As $w=0$ on $\Omega \backslash \Omega_{0}$ and $\|w\|_{\infty}=1,\left.w\right|_{\Omega_{0}}$ is nonnegative and not identically zero. Hence we must have $a=\lambda_{1}^{\Omega_{0}}$, contradicting our assumption that $a<\lambda_{1}^{\Omega_{0}}$. This proves our claim that $\left\{\alpha_{n}\right\}$ is bounded.

The rest of the proof follows that of Theorem 1.1 except that to prove $u_{p} \geq \beta U_{\alpha}$, we use Lemma 2.1 of [DM] (which holds for $C^{1}$ functions).

\section{Proof of Theorem 1.6.}

It turns out that Lemma 1.4 is not enough for our proof of Theorem 1.6. We will need some fine properties of the limiting function $u$ in Lemma 1.4 and of functions in $H^{1}\left(R^{N}\right)$. These fine properties have already been used in [DD2] and we simply collect them in the following lemma:

Lemma 5.1. Let $u$ and $u_{n}$ be as in Lemma 1.4. Then the following conclusions hold:

(i) $\widetilde{u}(x)=\lim _{r \rightarrow 0} \int_{B_{r}(x)} u(y) d y /\left|B_{r}(x)\right|$ exists for each $x \in \Omega$, where $B_{r}(x)$ denotes the ball with center $x$ and radius $r$, and $\left|B_{r}(x)\right|$ stands for the volume of $B_{r}(x)$. Moreover, $u=\widetilde{u}$ a.e. in $\Omega$.

(ii) $\widetilde{u}$ is upper semi-continuous (u.s.c. for short) on $\Omega$, and for each $x_{0} \in \Omega$ and any given $\epsilon>0$, we can find a small ball $B_{r}\left(x_{0}\right) \subset \Omega$ such that for all large $n$,

$$
u_{n}(x) \leq \widetilde{u}\left(x_{0}\right)+\epsilon, \forall x \in B_{r}\left(x_{0}\right) .
$$

(iii) If $v \in H^{1}\left(R^{N}\right)$, then $\widetilde{v}(x)=\lim _{r \rightarrow 0} \int_{B_{r}(x)} v(y) d y /\left|B_{r}(x)\right|$ exists for all $x \in R^{N}$ except possibly for a set of $(1,2)$-capacity 0 . Moreover, $\widetilde{v}=v$ a.e. in $R^{N}$ and if $\widetilde{v}$ vanishes on a closed set $A$ in $R^{N}$ (except for a subset of $A$ of capacity zero), then there exists a sequence of functions 
$\phi_{n} \in H^{1}\left(R^{N}\right)$ such that each $\phi_{n}$ vanishes in a neighbourhood of $A$ and $\phi_{n} \rightarrow \widetilde{v}$ in $H^{1}\left(R^{N}\right)$.

Let us now come back to the proof of Theorem 1.6. Let $p_{n}$ be a sequence converging to $\infty$ and use the notations in (2.2). Then as before, by Lemma 1.4 , subject to a subsequence, $w_{n} \rightarrow w$ weakly in $H_{0}^{1}(\Omega)$ and strongly in $L^{q}(\Omega)$ for any $q \geq 1$, and $\|w\|_{\infty}=1$.

Claim $1 .\left\{M_{n}\right\}$ is bounded.

Proof. Since $a<\lambda_{1}^{\Omega_{0}}$, we can find a small $\delta$-neighborhood $\Omega_{\delta}$ of $\bar{\Omega}_{0}$ such that $a<\lambda_{1}^{\Omega_{\delta}}$. Let $\phi_{\delta}$ denote the normalized positive eigenfunction corresponding to $\lambda_{1}^{\Omega_{\delta}}$ :

$$
-\Delta \phi_{\delta}=\lambda_{1}^{\Omega_{\delta}} \phi_{\delta},\left.\phi_{\delta}\right|_{\partial \Omega_{\delta}}=0,\left\|\phi_{\delta}\right\|_{\infty}=1,
$$

and let $\psi \in C^{2}(\bar{\Omega})$ be an extension of $\left.\phi_{\delta}\right|_{\Omega_{\delta / 2}}$ to $\bar{\Omega}$ such that $\eta:=\min _{\bar{\Omega}} \psi>0$. We find, for any positive constant $Q$,

$$
\begin{aligned}
& \Delta(Q \psi)+a(Q \psi)-b(Q \psi)^{p} \leq\left(a-\lambda_{1}^{\Omega_{\delta}}\right) Q \psi<0, \forall x \in \Omega_{\delta / 2}, \\
& \Delta(Q \psi)+a(Q \psi)-b(Q \psi)^{p}=Q(\Delta \psi+a \psi)-b Q^{p} \psi^{p}, \forall x \in \Omega \backslash \Omega_{\delta / 2} .
\end{aligned}
$$

Let $\xi=\inf _{\Omega \backslash \Omega_{\delta / 2}} b$ and

$$
Q_{p}:=\left[\xi^{-1} \sup _{\Omega}(\Delta \psi+a \psi) \eta^{-p}\right]^{1 /(p-1)} .
$$

We easily see that for $Q=Q_{p}$,

$$
\Delta(Q \psi)+a(Q \psi)-b(Q \psi)^{p} \leq 0, \forall x \in \Omega .
$$

Therefore $Q_{p} \psi$ is an upper solution of (1.1). As (1.1) has arbitrarily small positive lower solutions, its unique positive solution $u_{p}$ must satisfy $u_{p} \leq$ $Q_{p} \psi$. Clearly $Q_{p} \rightarrow 1 / \eta$ as $p \rightarrow \infty$. Thus, for any $p_{0}>1,\left\{M_{p}: p \geq p_{0}\right\}$ is bounded. In particular, $\left\{M_{n}\right\}$ is bounded. This proves Claim 1.

By passing to a subsequence, we may assume that $M_{n} \rightarrow c \in[0, \infty)$ as $n \rightarrow \infty$.

Claim 2. $c \geq 1$.

Proof. Let $v_{n}$ be the unique solution of

$$
-\Delta v=a v-\|b\|_{\infty} v^{p_{n}},\left.v\right|_{\partial \Omega}=0 .
$$

By Theorem 1.3 we know $\left\|v_{n}\right\|_{\infty} \rightarrow 1$. On the other hand, a simple comparison argument shows $u_{n} \geq v_{n}$. Hence $c \geq 1$.

Claim 3. $w \leq 1 /$ c a.e. in $\Omega \backslash \Omega_{0}$. 
Proof. Otherwise the set $\left\{x \in \Omega \backslash \Omega_{0}: w(x)>1 / c\right\}$ has positive measure and we can find some $c_{1}>1 / c$ such that $\Omega_{1}:=\left\{x \in \Omega \backslash \Omega_{0}: w(x) \geq c_{1}\right\}$ has positive measure. As $w_{n} \rightarrow w$ in $L^{2}(\Omega)$, by passing to a subsequence, $w_{n} \rightarrow w$ a.e. in $\Omega$. Hence, by Egorov's theorem, we can find a subset of $\Omega_{1}$, say $\Omega_{2}$ which has positive measure and such that $w_{n} \rightarrow w$ uniformly on $\Omega_{2}$. It follows that $u_{n} \rightarrow c w$ uniformly on $\Omega_{2}$. Thus, there exists $\epsilon>0$ such that for all large $n, u_{n} \geq 1+\epsilon$ on $\Omega_{2}$.

Let $\phi \in C_{0}^{\infty}(\Omega)$ be an arbitrary nonnegative function, and multiply the equation for $w_{n}$ by $\phi$ and integrate over $\Omega$. It results

$$
\int_{\Omega} w_{n}(-\Delta \phi)=a \int_{\Omega} w_{n} \phi-\int_{\Omega} b u_{n}^{p_{n}-1} w_{n} \phi
$$

Hence, for all large $n$,

$$
(1+\epsilon)^{p_{n}-1} \int_{\Omega_{2}} b w_{n} \phi \leq \int_{\Omega_{2}} b u_{n}^{p_{n}-1} w_{n} \phi \leq \int_{\Omega} w_{n}(\Delta \phi)+a \int_{\Omega} w_{n} \phi .
$$

Dividing the above inequality by $(1+\epsilon)^{p_{n}-1}$ and letting $n \rightarrow \infty$, we deduce

$$
\int_{\Omega_{2}} b w \phi=0
$$

It follows that $w=0$ a.e. in $\Omega_{2}$, contradicting the assumption that $w \geq c_{1}$ there. This proves Claim 3.

Using $u_{n}=M_{n} w_{n}$ and denoting $\hat{u}=c w$, we see from Lemma 5.1 and Claims 1-3 above that the following result holds:

\section{Lemma 5.2.}

(i) $\left\{\left\|u_{n}\right\|_{\infty}\right\}$ is bounded.

(ii) Subject to a subsequence, $u_{n} \rightarrow \hat{u}$ weakly in $H_{0}^{1}(\Omega)$ and strongly in $L^{q}(\Omega), \forall q \geq 1$.

(iii) $\hat{u} \leq 1$ a.e. in $\Omega \backslash \Omega_{0}$ and $\|\hat{u}\|_{\infty} \geq 1$.

(iv) $\widetilde{u}(x):=\lim _{r \rightarrow 0} \int_{B_{r}(x)} \hat{u}(y) d y /\left|B_{r}(x)\right|$ exists for every $x \in \Omega$.

(v) $\widetilde{u}(x)$ is u.s.c. on $\Omega$ and $\widetilde{u}=\hat{u}$ a.e. in $\Omega$.

(vi) For each $x_{0} \in \Omega$ and any given $\epsilon>0$, we can find a small ball $B_{r}\left(x_{0}\right) \subset$ $\Omega$ such that for all large $n$,

$$
u_{n}(x) \leq \widetilde{u}\left(x_{0}\right)+\epsilon, \forall x \in B_{r}\left(x_{0}\right) .
$$

We are now ready to complete the proof of Theorem 1.6. Multiplying the equation for $u_{n}$ by $\phi \in C_{0}^{\infty}(\Omega)$, we deduce

$$
\int_{\Omega} \nabla u_{n} \cdot \nabla \phi d x=a \int_{\Omega} u_{n} \phi d x-\int_{\Omega} b(x) u_{n}^{p_{n}} \phi d x .
$$

It follows that, subject to a subsequence,

(5.1) $\lim _{n \rightarrow \infty} \int_{\Omega} b(x) u_{n}^{p_{n}} \phi d x=-\int_{\Omega} \nabla \hat{u} \cdot \nabla \phi d x+a \int_{\Omega} \hat{u} \phi d x, \forall \phi \in C_{0}^{\infty}(\Omega)$. 
Clearly the right-hand side of (5.1) defines a continuous linear functional on $H^{1}(\Omega)$ :

$$
T(\phi)=-\int_{\Omega} \nabla \hat{u} \cdot \nabla \phi d x+a \int_{\Omega} \hat{u} \phi d x .
$$

Using the left-hand side of (5.1), and noticing that $b=0$ on $\Omega_{0}$, we see that $T(\phi) \geq 0$ whenever $\phi \in C_{0}^{\infty}(\Omega)$ satisfies $\phi \geq 0$ on $\Omega \backslash \Omega_{0}$. Moreover, if $\operatorname{supp}(\phi) \subset\{\widetilde{u}<1\} \cup \Omega_{0}$, where $\{\widetilde{u}<1\}:=\{x \in \bar{\Omega}: \widetilde{u}(x)<1\}$, then by Lemma 5.2 (vi) and the fact that $\{\widetilde{u}<1\}$ is relatively open (due to $\widetilde{u}$ being u.s.c), we can find $\delta>0$ such that $u_{n}(x) \leq 1-\delta$ on the compact set $\operatorname{supp}(\phi) \backslash \Omega_{0} \subset \operatorname{supp}(\phi) \cap\{\widetilde{u}<1\} \subset \Omega$ for all large $n$. Therefore, since $b=0$ on $\Omega_{0}$

$$
0 \leq \int_{\Omega} b(x) u_{n}^{p_{n}} \phi d x \leq \int_{\operatorname{supp}(\phi) \backslash \Omega_{0}} b(x)(1-\delta)^{p_{n}} \phi d x \rightarrow 0 .
$$

It follows that $T(\phi)=0$ if $\operatorname{supp}(\phi) \subset\{\widetilde{u}<1\} \cap \Omega_{0}$. Using the continuity of $T$ on $H^{1}(\Omega)$ and the fact that functions in $H_{0}^{1}(\Omega)$ can be approximated in the $H^{1}(\Omega)$ norm by functions in $C_{0}^{\infty}(\Omega)$, we find that

$$
\begin{aligned}
& T(\phi) \geq 0, \forall \phi \in H_{0}^{1}(\Omega) \text { satisfying } \phi \geq 0 \text { a.e. on } \Omega \backslash \Omega_{0}, \\
& T(\phi)=0, \forall \phi \in H_{0}^{1}(\Omega) \text { satisfying } \operatorname{supp}(\phi) \subset \Omega_{0} \cup\{\hat{u}<1\} .
\end{aligned}
$$

By Lemma 5.2 (iii), we easily see that $\widetilde{u} \leq 1$ on the open set $\Omega \backslash \bar{\Omega}_{0}$. We show next that $\widetilde{u}$ is close to 0 near $\partial \Omega$ and $\widetilde{u} \leq 1$ on $\partial \Omega_{0}$. By Lemma 5.2 (i), we can find $M>0$ such that $a u_{n}<M$ on $\Omega$ for all $n \geq 1$. Therefore

$$
-\Delta u_{n}=a u_{n}-b(x) u_{n}^{p_{n}} \leq M \text { on } \Omega \text {. }
$$

If $V$ is given by

$$
-\Delta V=M \text { in } \Omega,\left.V\right|_{\partial \Omega}=0,
$$

we obtain by the maximum principle that $u_{n} \leq V$. It follows that $\widetilde{u} \leq V$. Therefore $\widetilde{u}$ is close to 0 near $\partial \Omega$.

Since $\widetilde{u} \leq 1$ on $\Omega \backslash \bar{\Omega}_{0}$, we must have $\widetilde{u} \leq 1$ on $\partial \Omega_{0}$ except possibly for a set of capacity zero (see, e.g., [Z] pp. 190-191).

From the above analysis, we see that it is possible to choose $\phi \in C_{0}^{\infty}(\Omega)$ such that $0 \leq \phi \leq 1$ on $\Omega$ and $\phi=1$ on a $\delta$-neighborhood $N_{\delta}$ of $\{\hat{u}=1\}$. Let $v \in K$ be arbitrary and denote $\hat{v}=\max \{v, \phi\}$. Clearly $0 \leq \hat{v}-v \in H_{0}^{1}(\Omega)$. Thus, by (5.2),

$$
\begin{aligned}
& \int_{\Omega} \nabla \hat{u} \cdot \nabla(v-\hat{u}) d x-a \int_{\Omega} \hat{u}(v-\hat{u}) d x=-T(v-\hat{u}) \\
& =T(\hat{v}-v)+T(\hat{u}-\hat{v}) \geq T(\hat{u}-\hat{v}) .
\end{aligned}
$$

Denote $u^{*}=\hat{u}-\hat{v}$. Clearly $u^{*} \in H_{0}^{1}(\Omega)$. Now we choose $\psi \in C_{0}^{\infty}(\Omega)$ satisfying $0 \leq \psi \leq 1$ on $\Omega, \psi=0$ on $\Omega \backslash N_{(2 / 3) \delta}, \psi=1$ on $N_{(1 / 3) \delta}$. Then 
clearly

$$
\operatorname{supp}\left((1-\psi) u^{*}\right) \subset \bar{\Omega} \backslash N_{(1 / 3) \delta} \subset\{\widetilde{u}<1\} \cup \Omega_{0} .
$$

Hence, by (5.3),

$$
T\left(u^{*}\right)=T\left((1-\psi) u^{*}\right)+T\left(\psi u^{*}\right)=T\left(\psi u^{*}\right) .
$$

As $\psi=0$ on $\Omega \backslash N_{(2 / 3) \delta}$, and $\hat{v}=\max \{v, \phi\}=1$ a.e. on $N_{\delta}$, we find that $\psi u^{*}=\psi(\hat{u}-1)$ a.e. on $\Omega$. Since $\psi(\hat{u}-1)$ is zero outside $N_{(2 / 3) \delta}$ it can be regarded as a member of $H^{1}\left(R^{N}\right)$. It is easily seen that the representative of $\psi(\hat{u}-1)$ obtained through the limiting process in Lemma 5.1 (iii) is $\psi(\widetilde{u}-1)$. Thus we obtain

$$
T\left(u^{*}\right)=T\left(\psi u^{*}\right)=T(\psi(\widetilde{u}-1)) .
$$

As $\widetilde{u} \leq 1$ on $\bar{\Omega} \backslash \Omega_{0}$ and is u.s.c., we find that the set $A_{1}:=\{\widetilde{u}=1\} \cap\left(\bar{\Omega} \backslash \Omega_{0}\right)$ is closed. Let $A_{2}:=R^{N} \backslash N_{(2 / 3) \delta}$ and $A=A_{1} \cup A_{2}$. We know that $\psi(\widetilde{u}-1)$ vanishes on the closed set $A$ (except possibly for a set of capacity zero) and so by Lemma 5.1 (iii), it can be approximated in the $H^{1}\left(R^{N}\right)$ norm by $\phi_{n} \in H^{1}\left(R^{N}\right)$ with each $\phi_{n}$ vanishing in a neighbourhood of $A$. Therefore, $\operatorname{supp}\left(\phi_{n}\right) \subset\{\widetilde{u}<1\} \cup \Omega_{0}$, and by (5.3), $T\left(\phi_{n}\right)=0$. It follows that

$$
T\left(u^{*}\right)=T(\psi(\widetilde{u}-1))=\lim _{n \rightarrow \infty} T\left(\phi_{n}\right)=0 .
$$

We thus obtain

$$
\int_{\Omega} \nabla \hat{u} \cdot \nabla(v-\hat{u}) d x-a \int_{\Omega} \hat{u}(v-\hat{u}) d x \geq 0, \forall v \in K .
$$

That is to say that $\hat{u} \in K$ is a solution of (1.6). This finishes our proof of Theorem 1.6.

\section{References}

[AT] S. Alama and G. Tarantello, On the solvability of a semilinear elliptic equation via an associated eigenvalue problem, Math. Z., 221 (1996), 467-493, MR 97d:35067, Zbl 0853.35039.

[AM] P. Aviles and R. McOwen, Conformal deformation to constant negative scalar curvature on noncompact Riemannian manifolds, J. Diff. Geom., 27 (1988), 225239, MR 89b:58225, Zbl 0648.53021.

[D] E.N. Dancer, Some remarks on classical problems and fine properties of Sobolev spaces, Diff. Integral Eqns., 9 (1996), 437-446, MR 97e:35057, Zbl 0853.35011.

[DD1] E.N. Dancer and Y. Du, Effects of certain degeneracies in the predator-prey model, SIAM J. Math. Anal., 34 (2002), 292-316, CMP 1951776.

[DD2] - On a free boundary problem arising from population biology, Indiana Univ. Math. J., 52 (2003), 51-68.

[DD3] - A uniqueness theorem for a free boundary problem. Preprint, 2003.

[dP] M.A. del Pino, Positive solutions of a semilinear elliptic equations on a compact manifold, Nonlinear Analysis, 22 (1994), 1423-1430, MR 95g:58256, Zbl 0812.58077. 
[DH] Y. Du and Q. Huang, Blow-up solutions for a class of semilinear elliptic and parabolic equations, SIAM J. Math. Anal., 31 (1999), 1-18, MR 2000g:35059, Zbl 0959.35065.

[DM] Y. Du and L. Ma, Logistic type equations on $R^{N}$ by a squeezing method involving boundary blow-up solutions, J. London Math. Soc., 64 (2001), 107-124, MR 2002d:35089.

[FKLM] J.M. Fraile, P. Koch Medina, J. Lopez-Gomez and S. Merino, Elliptic eigenvalue problems and unbounded continua of positive solutions of a semilinear elliptic equation, J. Diff. Eqns., 127 (1996), 295-319, MR 97b:35036, Zbl 0860.35085.

[GT] D. Gilbarg and N.S. Trudinger, Elliptic Partial Differential Equation of Second Order, Springer-Verlag, 1977, MR 57 \#13109, Zbl 0361.35003.

$[\mathrm{KW}]$ J.L. Kazdan and F.W. Warner, Scalar curvature and conformal deformation of Riemannian structure, J. Diff. Geom., 10 (1975), 113-134, MR 51 \#1661, Zbl 0296.53037.

[M] L. Ma, Conformal deformations on a noncompact Riemannian manifold, Math. Ann., 295 (1993), 75-80, MR 93k:53040, Zbl 0788.58058.

[MV] M. Marcus and L. Veron, Uniqueness and asymptotic behavior of solutions with boundary blow-up for a class of nonlinear elliptic equations, Ann. Inst. H. Poincare Anal. Nonlineaire, 14 (1997), 237-274, MR 97m:35068, Zbl 0877.35042.

[O] T. Ouyang, On the positive solutions of semilinear equation $\Delta u+\lambda u-h u^{p}=$ 0 on the compact manifolds, Trans. Amer. Math. Soc., 331 (1992), 503-527, MR 92h:35012, Zbl 0759.35021.

[Z] W.P. Ziemer, Weakly Differentiable Functions, Springer-Verlag, New York, 1989, MR 91e:46046, Zbl 0692.46022.

Received July 2, 2001. The work of Dancer was partially supported by the ARC and partially done while visiting the Newton Institute at Cambridge, UK. Part of the work of $\mathrm{Du}$ and Ma was carried out while visiting the University of Sydney. Ma was partially supported by a grant from the National 973 Project of China and a scientific grant of Tsinghua University at Beijing.

School of Mathematics and Statistics

UNIVERSITY OF SYDNEY

NSW 2006

Australia

E-mail address: normd@maths.usyd.edu.au

School of Mathematical and Computer Sciences

UNIVERSITY OF NEW ENGLAND

ARMidale, NSW2351

Australia

E-mail address: ydu@turing.une.edu.au

Department of Mathematical Sciences

Tsinghua University

BEIJING 100084

CHINA

E-mail address: lma@math.tsinghua.edu.cn 